\title{
ACTIVIDAD ANTIOXIDANTE Y DETERMINACIÓN DE COMPUESTOS FENÓLICOS DEL CAIMITO (Pouteria caimito), CAIMITILLO (Chrsophylum sanguinolentum),GUAVA (Inga edulis) Y YARINA (Phytelephas macrocarpa)
}

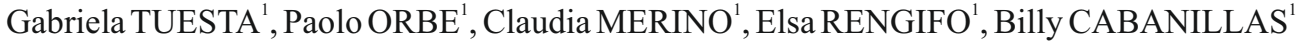 \\ 1 Laboratorio de Sustancias Naturales Bioactivas. Programa de Investigación en Biodiversidad Amazónica (PIBA). \\ Instituto de Investigaciones de la Amazonía Peruana-IIAP. Av. Abelardo Quiñones km 4.5, Iquitos, Perú. e-mail: \\ bcabanillas@iiap.org.pe.
}

\section{RESUMEN}

Se estudiaron frutos de caimito (Pouteria caimito), caimitillo (Chrsophylum sanguinolentum), guava (Inga edulis) y yarina (Phytelephas macrocarpa) para determinar su potencial antioxidante. Los extractos metanólicos de las cáscaras, pulpas y arilos fueron evaluados utilizando los métodos de ABTS y DPPH. Los resultados muestran que C. sanguinolentum poseen la mejor actividad antioxidante. Asimismo, se determinó el contenido total de compuestos fenólicos de los frutales estudiados, encontrándose que los valores obtenidos se correlacionan con la actividad antioxidante exhibida por las diferentes partes analizadas de los frutos.

PALABRAS CLAVE: actividad antioxidante, DPPH, ABTS, contenido de fenólicos totales, frutas amazónicas.

\section{ANTIOXIDANT ACTIVITY AND DETERMINATION OF PHENOLIC COMPOUNDS OF CAIMITO (Pouteria caimito), CAIMITILLO (Chrsophylum sanguinolentum), GUAVA (Inga edulis) Y YARINA (Phytelephas macrocarpa)}

\section{ABSTRACT}

The fruits of caimito (Pouteria caimito), caimitillo (Chrsophylum sanguinolentum), guava (Inga edulis) y yarina (Phytelephas macrocarpa) were analyzed to determinate their antioxidant activity. The methanolic extracts of the peel, pulp and aril were evaluated using the DPPH and ABTS methods. C. saguinolentum showed the best antioxidant activity employing both methods. Also, the total phenolic content was determinate and a correlation has been found between these results and the antioxidant activity exhibited by the fruits.

KEYWORDS: antioxidant activity, DPPH, ABTS, total phenolic content, Amazonian fruits. 


\section{INTRODUCCIÓN}

La oxidación es un proceso que involucra la transferencia de electrones de un átomo a otro, desarrollándose continuamente dentro el organismo humano. Sin embargo, cuando la transferencia es solo parcial, uno de los átomos queda con un solo electrón, generándose lo que se conoce como radical libre, una especie muy oxidativa y que reaccionará rápidamente con células cercanas provocándoles daño. El exceso de estas especies oxidativas, debido a fuentes internas y/o externas, es conocido como stress oxidativo, el cual está asociado a la aparición de muchas enfermedades como arterioesclerosis, cáncer, insuficiencia renal aguda y crónica, diabetes e hipertensión arterial, etc. (Young \& Woodside, 2001; Elejalde-Guerra, 2001). Todos los organismos aeróbicos poseen mecanismos de defensa que permiten remover o reparar las células dañadas. También contribuyen a estos procesos los compuestos antioxidantes que son ingeridos en los alimentos.

Durante los últimos años ha surgido un gran interés por la búsqueda de antioxidantes de origen natural, especialmente aquellos que están presentes en las plantas, debido principalmente a que el consumo de vegetales y frutas ha sido asociado con la disminución del riesgo de padecer enfermedades provocadas por el estrés oxidativo (Gülçin, 2012). Dentro de los grupos de compuestos presentes en estos alimentos e incluidos en la dieta humana diaria, destacan: la vitamina $\mathrm{C}$, los tocoferoles, los carotenoides y los flavonoides. En el mercado también existen antioxidantes sintéticos como el butilhidroxianisol (BHA), el butilhidroxitolueno (BHT), la terbutil hidroquinona (TBHQ) y el galato de propilo, que son empleados en alimentos y en medicamentos. Sin embargo, el uso de BHA y el BHT ha sido cuestionado debido a dudas sobre sus efectos tóxicos y carcinogénicos (Wichi 1988; Sherwin 1990).

El objetivo del presente estudio es evaluar la actividad antioxidante de cuatro frutales amazónicos sin estudios previos, con la finalidad de identificar aquellos que puedan ser incluidos con mayor frecuencia en la dieta diaria y de esta manera proteger la salud de la población mediante la disminución de las enfermedades generadas por el estrés oxidativo.

\section{MATERIALES Y MÉTODOS}

\section{Material vegetal}

Las frutos de Chrsophylum sanguinolentum, Pouteria caimito, Inga edulis y Phytelephas macrocarpa, fueron colectados del Centro de Investigaciones de Allpahuayo (CIA) ubicado en el $\mathrm{Km} 27,5$ de la carretera Iquitos-Nauta, provincia de Maynas, departamento de Loreto. Las determinaciones estuvieron a cargo de la bióloga Elsa Rengifo Salgado.

\section{Preparación de los extractos de frutos}

Para las muestras de los frutos, se pesaron 500 $\mathrm{mg}$ de las mismas (pulpa, cáscara o arilo) en un microtubo, se le agregaron $1,5 \mathrm{~mL}$ de metanol al $90 \%$, agitándose en un vortex durante 5 minutos. La mezcla se centrifugó a $5000 \mathrm{rpm}$ a $5^{\circ} \mathrm{C}$ durante 15 minutos. El residuo fue tratado nuevamente con 750 $\mu \mathrm{L}$ de metanol al $90 \%$. Ambas soluciones fueron unidas, llevadas en una fiola a $5 \mathrm{~mL}$ y guardadas a $20^{\circ} \mathrm{C}$ hasta su empleo.

\section{Ensayo de captura del radical dpph}

La actividad antioxidante de las muestras se determinó utilizando el reactivo DPPH (2,2- Difenil1-picrilhidrazilo), según la metodología descrita por Blois (1958) y revisada por Sharma \& Bhat (2009). Se prepararon las curvas de calibración de los patrones de actividad antioxidante conocidas (ácido ascórbico, quercetina y Trolox). La capacidad antioxidante de los patrones de los frutos fue expresada como equivalentes de Trolox (TEAC) y la concentración de antioxidante requerida para capturar el $50 \%$ de los radicales DPPH $\mathrm{EC}_{50}$, fue calculada mediante regresión lineal, utilizando el programa Excel. Todos los experimentos fueron realizados por triplicado.

\section{Ensayo de la captura del radical abts}

La actividad antioxidante, que fue medida utilizando el reactivo ABTS (ácido 2,2' 'azino-bis(3etilbenzotiazolin)-6-sulfónico), también fue expresada como actividad antioxidante expresada en equivalentes de Trolox (TEAC). La metodología utilizada fue la propuesta por Rice Evans et al. (1999). El radical ABTS ${ }^{+}$fue preparado por reacción entre el ABTS $(7 \mathrm{mM})$ y el persulfato de potasio (2.45 mM) durante $16 \mathrm{~h}$ a $25^{\circ} \mathrm{C}$ y al abrigo de la luz. Una vez formado el radical $\mathrm{ABTS}^{+}$se diluyó utilizando un buffer PBS $0,01 \mathrm{M}(\mathrm{pH} 7,4)$ hasta alcanzar una absorbancia de $0,70( \pm 0,10)$ a $754 \mathrm{~nm}$. Se prepararon diluciones del extracto de manera que se pudiera producir inhibiciones del radical entre el $20-80 \%$. El $\mathrm{EC}_{50}$ fue calculado mediante regresión lineal empleando el programa Excel. Los experimentos se condujeron por triplicado. 


\section{Determinación del contenido \\ Total de fenólicos}

La cantidad total de fenólicos presentes en las diferentes frutas fue determinada empleando el reactivo de Folin-Ciocalteu, siguiendo el procedimiento descrito por Singleton et al. (1965). Los resultados fueron expresados como equivalentes de ácido gálico (GAE) en mg por $100 \mathrm{~g}$ de muestra fresca.

\section{RESULTADOS Y DISCUSIONES}

La complejidad de la mezcla de compuestos en un extracto, muchas veces requiere de condiciones especiales para poder medir la actividad antioxidante, por esta razón existen varios métodos para la determinación de la capacidad antioxidante (Prior et al., 2005). Además, el empleo de varias técnicas para evaluar la actividad antioxidante permite obtener mayor grado de información para conocer más acerca del comportamiento de los extractos. En el presente estudio se ha evaluado la actividad antioxidante de los frutos de Chrsophylum sanguinolentum, Pouteria caimito, Inga edulis y Phytelephas macrocarpa, utilizando dos métodos estandarizados: el ensayo de captura del radical DPPH y la prueba de captura del radical ABTS. Además, se midió la actividad antioxidante del ácido ascórbico, trolox y quercetina, tres antioxidantes comunes utilizados como estándares para comparar el potencial antioxidante de los frutales evaluados.

La concentración eficaz media $\left(\mathrm{EC}_{50}\right)$ es la concentración de extracto necesaria para poder neutralizar la mitad de radicales libres presentes en la solución; cuanto menor sea el valor de $\mathrm{EC}_{50}$, el extracto probado poseerá una mayor capacidad para capturar (o neutralizar) los radicales libres y por ende tendrá una mejor actividad antioxidante.

Los valores de $\mathrm{EC}_{50}$ para las especies estudiadas, empleando el método el DPPH, se muestran en la Tabla 1. La cáscara y la pulpa del caimitillo $(C$. sanguinolentum) exhibieron una muy buena actividad antioxidante con valores de $\mathrm{EC}_{50}$ de 17,54 y $19,98 \mathrm{mg} \cdot \mathrm{mL}^{-1}$, respectivamente; el arilo arrojó una actividad moderada $\left(\mathrm{EC}_{50}=81,54 \mathrm{mg} \cdot \mathrm{mL}^{-1}\right)$. Los valores mostrados por la cáscara y la pulpa fueron cercanos a aquellos mostrados por los estándares del ácido ascórbico, Trolox y quercetina, lo que sugiere que estas partes del fruto poseen un alto contenido de compuestos antioxidantes. Por otro lado, la cáscara del caimito ( $P$. caimito) solo exhibió una actividad moderada, y la pulpa una actividad muy débil $\left(\mathrm{EC}_{50}>\right.$ $\left.1000 \mathrm{mg} \cdot \mathrm{mL}^{-1}\right)$. Asimismo, la pulpa de la yarina $(P$. macrocarpa) también exhibió una actividad muy baja en la captura de los radicales DPPH. El arilo de la guaba (I. edulis) mostró una moderada actividad antioxidante $\left(\mathrm{EC}_{50}=105,74 \mathrm{mg} \cdot \mathrm{mL}^{-1}\right)$, pero inferior a aquella exhibida por el arilo del caimito.

Una tendencia similar se observó cuando se midió la actividad antioxidante empleando el ABTS (Tabla 1). La cáscara y pulpa del caimitillo $(C$. sanguinolentum) mostrarón la mayor actividad. Por otro lado, la baja actividad exhibida por la cáscara y la pulpa del caimitio (P. caimito) en la prueba con el DPPH mejoró considerablemente cuando se empleó el ABTS. El extracto del arilo de la guaba (I. edulis) exhibió una actividad similar a la pulpa de la yarina (P. macrocarpa), que mostró una actividad antioxidante débil $\left(\mathrm{EC}_{50}=412,46 \mathrm{mg} \cdot \mathrm{mL}^{-1}\right)$ al igual que cuando se empleó el DPPH.

Las diferencias encontradas en los valores de actividad al realizar ambos métodos, también fueron encontradas en trabajos previos (Floegel et al., 2011), donde se indica que es con el ABTS con el que se suelen obtener mejores valores de actividad y en algunos casos muy diferenciados a la de aquellos valores obtenidos empleando el DPPH, especialmente debido a que la absorción a $734 \mathrm{~nm}$ del ABTS elimina las interferencias de color ( $\mathrm{Li}$ et al., 2008). También se conoce que la prueba con ABTS es la que mejor se correlaciona con los resultados que genera la prueba ORAC (Capacidad de absorbancia del radical oxígeno), una prueba muy empleada para medir la actividad antioxidante sobre algunas especies oxigenadas.

Los compuestos fenólicos son uno de los grupos de compuestos más abundantes en las plantas, muchos de ellos han mostrado múltiples efectos biológicos, incluyendo actividad antioxidante (Kähkönen et al., 1999). Como parte de la evaluación de la actividad antioxidante de los frutales en estudio, se determinó el contenido de compuestos fenólicos, con la finalidad de estudiar la posible asociación entre la cantidad de estos compuestos y la actividad antioxidante mostrada de cada una de las frutas estudiadas. Los resultados muestran una correlación entre el contenido de compuestos fenólicos y la actividad antioxidante de los frutales analizados (Tabla 1). De esta forma, el mayor contenido de compuestos fenólicos corresponde al caimitillo, el compuesto que mostró la mejor actividad antioxidante tanto con el DPPH como con el ABTS, en especial para la cáscara $\left(995,23 \mathrm{mg} \mathrm{AG} / 100 \mathrm{~g} \mathrm{MF}, \mathrm{EC}_{50 \mathrm{ABTS}}=16,34 \mathrm{mg} / \mathrm{mL}\right)$ y la pulpa $\left(392,49 \mathrm{mg} \mathrm{AG} / 100 \mathrm{~g} \mathrm{MF}, \mathrm{EC}_{50 \mathrm{ABTS}}=26,47\right.$ $\mathrm{mg} / \mathrm{mL}$ ). Un comportamiento similar se observó cuando se compararon los valores obtenidos para el caimito, la guaba y la yarina. 
Tabla 1. Actividad antioxidante determinada mediante DPPH y ABTS y contenidos de fenólicos totales de cuatro frutas amazónicas

\begin{tabular}{lccc}
\hline \multicolumn{1}{c}{ Muestra } & $\begin{array}{c}\mathbf{D P P H} \\
\mathbf{E C}_{50}(\mathbf{m g} / \mathbf{m L})\end{array}$ & $\begin{array}{c}\text { ABTS } \\
\mathbf{E C}_{50}(\mathbf{m g} / \mathbf{m L})\end{array}$ & $\begin{array}{c}\text { Fenólicos Totales } \\
\mathbf{m g ~ A G} / \mathbf{~ 1 0 0 g ~ M F ~}\end{array}$ \\
\hline C. sanguinolentum (cáscara) & 17,54 & 16,34 & 995,23 \\
C. sanguinolentum (pulpa) & 20,73 & 26,47 & 392,49 \\
C. sanguinolentum (arilo) & 81,54 & 93,68 & 76,84 \\
\hline P. caimito (cáscara) & 242,29 & 27,76 & 20,82 \\
\hline P. caimito (pulpa) & $>1000$ & 74,20 & 7,81 \\
\hline I. edulis (arilo) & 105,74 & 192,39 & 24,61 \\
P. macrocarpa (pulpa) & $>1000$ & 412,46 & 8,70 \\
Acido ascórbico & $3,07(17,44 \mu \mathrm{M})$ & $11,70(66,43 \mu \mathrm{M})$ & - \\
Quercetina & $4,28(14,16 \mu \mathrm{M})$ & $4,74(15,67 \mu \mathrm{M})$ & - \\
\hline Trolox & $4,47(17,84 \mu \mathrm{M})$ & $9,74(38,93 \mu \mathrm{M})$ & - \\
\hline
\end{tabular}

\section{CONCLUSIONES}

La evaluación de la actividad antioxidante de cuatro frutales amazónicos: Chrsophylum sanguinolentum, Pouteria caimito, Inga edulis y Phytelephas macrocarpa permitió determinar que el caimitillo (C. sanguinolentum) posee un alto potencial antioxidante y que este depende principalmente del importante contenido de compuestos fenólicos. El caimito solo posee una actividad antioxidante moderada, mientras que los frutos de I. edulis y P. macrocarpa analizados poseen una baja actividad antioxidante. Con estos resultados, se puede sugerir que la inclusión en la dieta diaria del caimito y del caimitillo ayudaría a prevenir las enfermedades generadas por el estrés oxidativo.

La cáscara y la pulpa del caimitillo poseen la mejor actividad antioxidante, siendo las partes normalmente más consumidas del fruto, por lo que su empleo en la industria de alimentos estaría orientado sobre todo a sus propiedades como preservante.

\section{BIBLIOGRAFÍA}

Blois, M.S. 1958. Antioxidant determinations by the use of a stable free radical. Nature, 181: 11991200.
Elejalde-Guerra, J.J. 2001. Estrés oxidativo, enfermedades y tratamientos antioxidantes. Anales de Medicina Interna, 18 (6): 326-335.

Floegel, A.; Kim,D-O.; Chung, S-J.; Koo, S.I.; Chun, O.K. 2011. Comparison of ABTS/DPPH assays to measure antioxidant capacity in popular antioxidant-rich US foods. Journal of Food Composition and Analysis, 24: 1043-1048.

Gülçin, I. 2012. Antioxidant activity of food constituents: an overview. Archives of Toxicology, 86: 345-391.

Halliwell, B. 1995. Antioxidant characterization. Methodology and mechanism. Biochemical Pharmacology, 49:1341-1348.

Kähkönen, M. P.; Hopia, A. I.; Vuorela, H. J.; Rauha, J.-P.; Pihlaja, K.; Kujala, T. S.; Heinonen, M. 1999. Antioxidant activity of plant extracts containing phenolic compounds. Journal of the Agricultural and Food Chemistry, 47: 39543962.

Li, H-B; Wonga, C-C; Chenga, K.; Chena, F. 2008. Antioxidant properties in vitro and total phenolic contents in methanol extracts from medicinal plants. LWT - Food Science and Technology, 41: 385-390.

Prior, R.L.; Wu, X. Schaich, K. 2005. Standardized Methods for the Determination of Antioxidant 
Capacity and Phenolics in Foods and Dietary Supplements. J Journal of the Agricultural and Food Chemistry, 53: 4290-4302.

Re, R.; Pellegrini, N.; Proteggente, A.; Pannala, A.; Yang, M.; Rice-Evans, C. 1999. Antioxidant activity applying an improved ABTS radical cation decolorization assay. Free Radical Biology \& Medicine, 26: 1231-1237.

Young, I.S.; Woodside J.V. 2001. Antioxidants in health and disease. Journal of Clinical Pathology, 54:176-186.

Sharma, O.P.; Bhat, T.K. 2009. DPPH antioxidant assay revisited. Food Chemistry, 113: 1202-1205.

Sherwin, E.R. (1990) In: Branen A.L., Davidson P.M., Salminen S. (eds), Food additives, Marvel Dekker Inc., New York, p 139-193.
Singleton, V.L.; Rossi, J.A. 1965. Colorimetry of Total Phenolics with PhosphomolybdicPhosphotungstic Acid Reagents. American Journal of Enology and Viticulture, 16: 144-158.

Wichi, H.P. 1988. Enhanced tumour development by butylated hydroxyanisole (BHA) from the perspective of effect on forestomach and oesophageal squamous epithelium. Food and Chemical Toxicology, 26: 717-723.

Recibido: 11/01/2014

Aceptado para publicación: 12/04/2014 
